\title{
Recognition of pictorial as compared with verbal descriptions*
}

\author{
STEPHEN A. BRUNETTE and WILLIAM F. BATTIG $\dagger$ \\ University of Colorado, Boulder, Colorado 80302
}

\begin{abstract}
After being presented with either photographs and/or sentential descriptions thereof, Ss were tested both for pictorial and verbal recognition both immediately and after $48 \mathrm{~h}$. Superior recognition was found for the type of material presented during study, primarily due to very poor pictorial recognition by Ss who had studied sentences. No decrement was found over the delay interval, which actually produced facilitation for the nonpresented modality.
\end{abstract}

The possible existence of separate perceptual and/or memorial processing systems for visual or imaginal, as distinguished from verbal or linguistic, types of information has become a major focus of recent research interest (e.g., Chase \& Clark, 1972; Paivio, 1971). In the present experiment, an attempt was made to explore possible similarities and differences in recognition performance under conditions where materials were presented for study and recognition testing in pictorial and/or verbal descriptive modes. In contrast with most previous research in this area, photographs taken in everyday naturalistic settings familiar to the present Ss were used as pictorial materials, along with verbal sentential descriptions thereof.

If pictorial and verbal modes are functionally separate, then initial presentation and processing in either mode should produce little, if any, transfer to recognition performance tested in the other mode. This possibility was directly evaluated in the present experiment by comparing recognition accuracy when tested in the opposite, as compared with the same mode, as had been used for presentation of the materials, with each $\mathrm{S}$ being tested on each item for both verbal and pictorial recognition.

A major problem complicating direct comparisons between pictorial and verbal modes is the virtual impossibility of equating pictures and verbal descriptions as to amount and kind of information contained therein. While the present research makes no pretense of having produced such equivalence, substantial efforts were made to minimize differences in informational content between pictorial and verbal descriptions. As described in more detail in the Method section, the present pictorial materials were color photographs with secondary and background details marked out on the

*This is publication No. 33 of the Institute for the Study of Intellectual Behavior, University of Colorado, and is based in part on an und ergraduate honors thesis by the first author. The research was also partially supported by Grants GB-25433 and GB-34077X from the National Science Foundation.

†Requests for reprints should be sent to William F. Battig, Institute for the Study of Intellectual Behavior, Muenzinger Building, University of Colorado, Boulder, Colorado 80302. basis of pretesting. Verbal descriptions of the essentials of each picture were then obtained from Ss asked to write sentential descriptions thereof. For purposes of the recognition tests, pairs of photographs were taken which were comparable in content except for one major difference between the two pictures of each pair. Each S was actually presented with only one of the two members of each picture or sentence pair, and subsequently tested for recognition by being presented both members of all pairs and responding to each individual picture or sentence by indicating whether it was "old" or "new." Such recognition tests both for pictures and sentences were given both immediately after presentation of the materials and after a 48-h delay. An additional experimental variable was presence or absence of an interfering task wherein Ss were required to count the number of vowels in a sentence or series of letters during the presentation of each picture or descriptive sentence.

\section{METHOD}

\section{Experimental Conditions and Materials}

Three major experimental conditions differed in type of material presented and responses made thereto prior to recognition testing. The picture-sentence group was presented with 28 unrelated pictures and asked to write a one-sentence description of each picture. The picture group was shown these same pictures but not required to write verbal descriptions thereof. Each $\mathrm{S}$ in the sentence group was shown 28 actual sentences written by Ss run previously in the picture-sentence group, and asked to write these sentences during their presentation. In addition to the tasks described above, half of the Ss in each group were also required, during each item presentation, to count and record numbers of vowels contained in the sentences, or (for the picture group) in nonsense letter strings equal in length to the corresponding sentences.

All materials were based upon a set of 56 color photographs taken in familiar natural settings and presented via Carousel slide projector. These pictures represented 28 pairs with the two pictures in each pair differing only in one specific way, e.g., the same man either reading a book or magazine, an arrow on a traffic sign pointing either left or right, a girl with either a black or white cat on her lap. The two pictures of each pair were arbitrarily divided into two sets of 28 each, each set being used 
equally often as the presentation and recognition distractor set (as described below).

\section{Procedure}

All Ss were initially presented one picture or descriptive sentence from each of the 28 pairs for $30 \mathrm{sec}$ each, during which time they studied the presented items, wrote or copied descriptive sentences, and counted vowels, as required by their experimental condition. Immediately after the last presented item, all Ss were given recognition tests in which all 56 pictures and the corresponding sentences for each were presented under self-paced conditions one at a time, with $S$ being required to label each picture and sentence as describing either an old previously presented or new unpresented item. For each picture and sentence, Ss were also required to indicate their confidence in the correctness of each response on a 6-point rating scale (which showed no group or condition effects of interest and thus will not be discussed further). No feedback about correctness of responses was ever given during or after the recognition tests. Half the Ss in each of the three groups (as well as each subgroup with and without the added vowel-counting task and with each of the two presented 28-item lists) were tested for the recognition of the 56 pictures prior to the 56 sentences, with the remaining Ss being tested for verbal prior to pictorial recognition. The same presentation order was used for all Ss and for both types of recognition tests, such that each of the 28 item pairs was represented once in each half of the recognition test order. This same recognition procedure was repeated after a 48 -h retention interval, using the same order of pictorial-verbal recognition as in the immediate test.

\section{Subjects}

The Ss were 64 introductory psychology students partially fulfilling a course requirement through their participation. Of the first $48 \mathrm{Ss}, 16$ each were randomly assigned to the picture-sentence, picture, and sentence groups, with $8 \mathrm{Ss}$ in each group required also to count vowels, and equal numbers within each group and subgroup getting each of the four possible combinations of the two 28-item presentation lists and verbal-pictorial or pictorial-verbal recognition test sequences. Subsequently it was found that $35 \%$ of the sentences produced by the picture-sentence group failed to include the critical feature distinguishing the two pictures of a given pair, which produced most of the recognition errors on pictures by the sentence group $(70 \%)$ and on sentences by the picture group $(80 \%)$. Thus 16 additional Ss were run subsequently, 8 each in the picture and sentence groups (equally divided among vowel-counting conditions, lists, and test sequences) all using the same 56 sentences which had been carefully selected to insure that each described adequately the critical feature distinguishing the two pictures within each pair.

\section{RESULTS}

Because all picture-sentence Ss were virtually perfect both in verbal (98.5\%) and pictorial recognition (96.9\%), only the data for the sentence and picture groups will be presented here. To correct for errors produced by inadequate sentence descriptions for the original $32 \mathrm{Ss}$ in the sentence and picture groups, adjusted percentage recognition scores for each of these Ss were based only upon those sentences which included the feature distinguishing the correct and incorrect members of a given pair. These adjusted scores for the original $32 \mathrm{Ss}$ were shown by analysis of variance not to differ significantly from the percentage recognition scores for the subsequent 16 Ss (who received only accurate sentences). Also no main effects nor interactions involving other major variables differed significantly in these recognition scores for the two sets of Ss. Thus, all data and analyses reported here are based upon all $24 \mathrm{Ss}$, both in the picture and sentence groups. Since neither the vowel-counting task nor the two presentation lists produced any significant effects upon recognition accuracy, either alone or in interaction with other major variables, both factors will be omitted from the discussion to follow.

As shown in the last column of Table 1, the picture group was markedly superior to the sentence group in overall recognition accuracy, $\mathrm{F}(1,32)=20.11, \mathrm{p}<.001$. This was due entirely, however, to the extremely poor (and insignificantly above chance) recognition of pictures by the sentence group. As shown in the second column of Table 1 , the sentence group actually showed slightly better verbal than pictorial recognition, producing a highly significant interaction of Type of Recognition by Groups $F(1,32)=137.40, p<.001$. The poor pictorial recognition by the sentence group also accounts for a significant overall superiority of verbal over pictorial recognition $\mathrm{F}(1,32)=21.56, \mathrm{p}<.00 \mathrm{i}$.

Also shown in Table 1 (by numbers in parenthesis) is a large improvement from immediate to delayed pictorial recognition by the sentence group, along with a lesser but still substantial immediate-delayed improvement in verbal recognition by the picture group. This contrasts sharply with the slight decrement in delayed recognition shown by both groups on the type of material originally presented. The consequent overall superiority for delayed over immediate recognition was significant $F(1,32)=9.76, p<.005$, but its three-way interaction was even larger $\mathrm{F}(1,32)=31.30, \mathrm{p}<.001$. Also significant was the Immediate-Delayed by Pictorial-Verbal interaction $\mathrm{F}(1,32)=4.95, \mathrm{p}<.05$.

Although the sequence in which pictorial and verbal recognition tests were given had no overall effect $(\mathrm{F}<1)$, the picture group showed better overall recognition when the initial test was pictorial $(91.8 \%)$ than verbal $(82.3 \%)$. Conversely, for the sentence group the pictorial-verbal sequence produced poorer recognition $(64.4 \%)$ than the verbal-pictorial sequence $(75.4 \%)$. This recognition superiority when the presented modality was tested first produced a

Table 1

Mean Percent Correct Pictorial and Verbal Recognition by Picture and Sentence Groups

\begin{tabular}{lccr}
\hline Group & Pictorial & Verbal & Total \\
\hline Picture & $92.78(-1.04)$ & $81.32(+5.51)$ & 87.05 \\
Sentence & $56.62(+12.35)$ & $83.11(-2.82)$ & 69.87 \\
Total & 74.70 & 82.22 & \\
\hline
\end{tabular}

Note-Differences for delayed minus immediate recognition in parentheses. 
significant interaction between groups and testing sequences $F(1,32)=7.18, p<.05$.

\section{DISCUSSION}

The present results offer some indication of separate pictorial and verbal processing systems, since recognition was consistently reduced when tested within a different modality from that used during presentation. As shown in Table 1, however, this effect was localized primarily in the extremely poor pictorial recognition by the sentence group, with verbal recognition being nearly as high for the picture as the sentence group.

One possible interpretation of this asymmetrical transfer between pictorial and sentential modes would be based upon the greater and more detailed information contained in the present pictures than sentences. However, Nelson, Metzler, and Reed (1974) report no facilitation of delayed recognition of pictures containing more detailed information. Alternatively, this greater transfer from pictures to sentences could reflect a greater likelihood that Ss will generate verbal representations of pictorial stimuli, and/or a closer correspondence or similarity of S's verbal description to a picture from which it was generated. Sentential stimuli, on the other hand, appear likely either to produce no image at all or else a relatively impoverished image which is too discrepant to be judged equivalent to the actual photograph from which this sentence was generated. Such an interpretation of course, would be equally consistent either with separate or identical processing systems for pictorial and verbal material

Also requiring explanation is the present marked improvement in 48-h delayed over immed iate recognition for the modality not used during presentation. Although no feedback was given during recognition testing, Ss nonetheless may have been able to use the presentation therein of exactly corresponding sets of pictures and sentences to improve their recognition accuracy especially for the modality used for the first time during the immediate recognition test. Such "learning" could also account for the better overall recognition shown when the presented modality was tested first in the recognition sequence, thus enhancing the availability of these presented stimuli to facilitate recognition also of the corresponding stimuli in the nonpresented modality.

\section{REFERENCES}

Chase, W. G., \& Clark, H. H. Mental o perations i $n$ the comparison of sentences and pictures. In L. W. Gregg (Ed.) Cognition in learning and memory. New York: Wi ley, 1972.

Telson, T O Metzler, J., \& Reed, D. A. Role of details in the long-term recognition of pictures and verbal descriptions. Journal of Experimental Psy chology, 1974, 102, 184-186.

Paivio, A. Imagery and verbal processes. New York: Holt, Rinehart, \& Winston, 1971.

(R eceived for publication August 5, 1974.)

\title{
Long-delay learning in rats: A black-white discrimination*
}

\author{
SAM REVUSKY \\ Memorial University, St. John's, Newfoundland, Canada
}

\begin{abstract}
Rats can associate between events separated by intertrial intervals as long as several hours. For instance, they can learn that the type of food reward on one trial in a runway is correlated with whether running will be rewarded on the following trial. They also can learn that they will be rewarded if they alternate responses in a T-maze. In the otherwise similar experiments reported here, more traditional discriminative stimuli were used. Whether running down a runway was to be rewarded with sugar solution depended on whether the goalbox on the preceding trial, over $4 \mathrm{~min}$ ago, had been white or black. The rats learned this discrimination and retained it through an extinction phase.
\end{abstract}

Animals made sick as much as several hours after feeding exhibit bait shyness. This association of feeding cues with delayed sickness can occur without isolation of the animal from the many extraneous cues which naturally occur during a delay; these include uncontrolled sounds and visual stimuli as well as the movements of the animal itself. Nor is there any direct or indirect temporal continguity between the feeding cues and sickness (Revusky \& Garcia, 1970). Underlying this learning is a mechanism called stimulus relevance:

* Research supported by National Research Council of Canada Grant A-8271. B. T. Lett and Harry Taukulis edited this paper. F. Chapman, C. Gan, E. Harley, and K. Winter trained the rats. feeding cues become associated with sickness far more readily than other cues (Capretta, 1961; Garcia, McGowan, \& Green, 1972). As a result, the extraneous cues bound to occur during a long delay do not confuse the animal and prevent learning.

These findings challenge the empiricistic theory that there are no innate criteria for selective association. The belief that temporal contiguity (or near contiguity) is necessary for learning is a corollary of any explanation of selective association by strict empiricistic principles; for empiricism admits to no other means of selective association between dissimilar events imbedded in the hundreds of events bound to affect an animal during a 